\title{
Software Engineering for Mapping Radio Frequency Pollution
}

\author{
Zenon Chaczko, Khalid Aboura and Johnson Agbinya
}

\begin{abstract}
Electromagnetic fields radiation has raised concerns within several segments of the population in the past three decades. Many studies proved inconclusive, in part due to the scarcity of data. We propose the idea of a geographical model based radiation pollution database. We discuss networked sensing technology for detection and monitoring of electromagnetic fields. We elaborate on software engineering issues for the visualization in real time of electromagnetic field mappings and the dissemination of information through various means and levels of access. We propose the database be complimented by a data algorithmic software solution for the extraction of patterns.
\end{abstract}

Keywords-Electromagnetic pollution, environmental monitoring, software engineering.

\section{INTRODUCTION}

$\mathbf{I}$ $\mathrm{N}$ recent times, Radio Frequency (RF) radiation emanating from wireless control and communication relays has become the subject of studies for possible links to health hazards, particularly cancer in children and adults. While no study has been accepted as conclusive evidence on the ill effects of Electromagnetic Fields (EMF) radiation, there have been a number of results urging caution in exposure to the electromagnetic waves. With the ever increasing deployment of wireless technology, more research at a larger scale will undoubtedly be undertaken on the subject of cause and effect of EMF radiation. A hindering aspect is the limitation in availability of radiation data over large areas such as cities, towns and inhabited farmlands. In this article, we discuss the need to develop the technology for a radio frequency mapping system that can be deployed over populated areas such as cities, industrial zones and inhabited places. We discuss the development of a data collection and visualization technology for the benefit of researchers, public agencies and commercial companies. The technology would benefit researchers from all parts of industries and governmental institutions and allow them to have easy access to reliable EMF mapping data. We briefly review existing work and propose an approach for radio frequency pollution mapping.

\section{Electromagnetic Fields Harmful EFFEcts}

The possibility that exposure to EMF radiation is associated with an increased risk of cancer has been debated since 1979

This work was supported by the Centre for Real-Time Information Networks (CRIN) of the University of Technology Sydney.

Z. Chaczko and J. Agbinya are with the School of Computing and Communications, University of Technology Sydney, 15 Broadway, U1timo, NSW 2007, Australia (e-mail: Zenon.Chaczko@uts.edu.au; Johnson.Agbinya@uts.edu.au).

K. Aboura is with the School of Civil and Environmental Engineering, University of Technology Sydney, 15 Broadway, Ultimo, NSW 2007, Australia (e-mail: Khalid.Aboura@uts.edu.au).

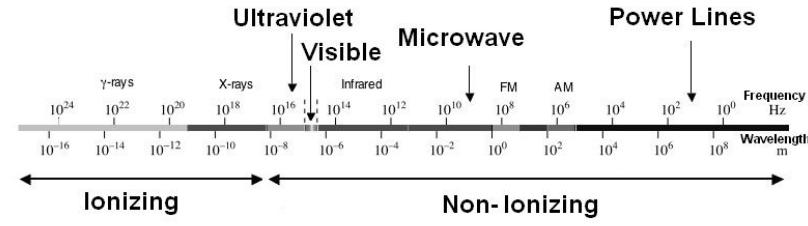

Fig. 1. The electomagnetic field spectrum.

when a study by Wertheimer and Leeper (1979) [30] appeared in the American Journal of Epidemiology. Initial fears focused on low frequency electromagnetic fields generated by electric currents in power lines and a large number of studies followed. A causal connection between EMF emanating from power lines and cancer could not be established. Many limitations were found in the studies conducted such as estimating the exposure to the EMF rather than directly measuring them. In 1997, the New England Journal of Medicine editorial called for a cessation of studies on the topic [5]. Electromagnetic fields from power lines are of extremely low frequency and are not believed to be powerful enough to cause biological damage. In addition, a number of animal studies found no convincing evidence of power frequency electromagnetic fields increasing the risk of cancer [21]. The US National Research Council spent several years reviewing hundreds of scientific studies conducted over a 20 year period and found "no conclusive and consistent evidence" that electromagnetic fields harm humans.

The interaction of biological matter with an electromagnetic source depends on the frequency of the source. The EMF spectrum consists of the magnetic fields from electric power systems, radio-frequency (RF) energy, microwaves (MW), infrared light (IR), visible light, ultraviolet (UV) light, X-rays and $\gamma$-rays (Figure 1). An EMF produces waves of energy that also act as particles. At high frequencies, these particle, called photons, can have pronounced biological effects. At the very high frequencies, less than 100 nanometers, electromagnetic particles have sufficient energy to break chemical bonds, a process called ionization. At lower frequencies, such as those characteristic of visible light and radio-frequencies, the energy of a photon is not enough to disrupt chemical bonds. Nonionizing electromagnetic sources in lower frequencies of the spectrum (Figure 1) produce known biological effects such as electron excitation (ultraviolet light, visible light, infrared light) and heating (microwaves and higher-frequency radio frequency energy). However, there is no doubt as to the severity of the damage that can be caused by the EMF in the ionizing part of the frequencies spectrum. In those cases, 


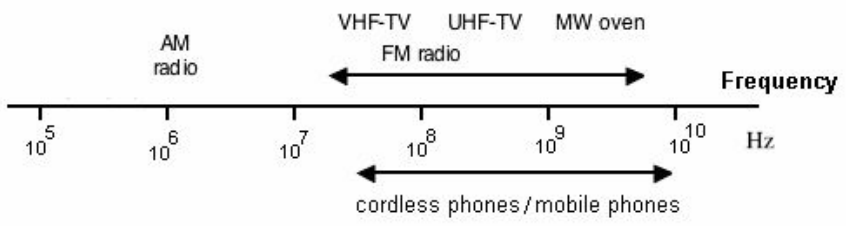

Fig. 2. The radio frequency spectrum.

radiation carries enough energy per photon to break bonds in the DNA, the genetic material of the cell. This can result in death, tissue damage and cancer. The controversy arises as to whether the non-ionizing part of the spectrum leads to severe health issues, including cancer.

Many studies rejected the hypothesis of a strong link between cancer and exposure to electromagnetic fields generated by power lines. For example, in 1996, a study by a group of Finnish researchers [28] found that typical residential magnetic fields generated by high voltage power lines do not seem to be related to the risk of overall cancer in adults. In 1999, the U.S. National Institute of Environmental Health Sciences issued a report to the U.S. Congress on "Health Effects from Exposure to Power-Line Electric and Magnetic Fields" [20]. The report concluded that there was no strong evidence to label extremely low frequency range electromagnetic fields exposure as a "known human carcinogen" or as a "probable human carcinogen".

While most scientists came to consider the evidence that power line fields can cause or contribute to cancer is weak to nonexistent, doubt remained as to whether EMF exposure was safe [25]. The awareness of the issue is heightened further with the constant introduction of new electronic devices in households and work places and an ever increasing number of wireless communication capabilities. These devices emit and receive within a frequency range of the EMF spectrum (Figure 2 ). Due to the non-ionizing nature and known heating quality of electromagnetic waves at some radio frequencies, until late 1996, most governments and all mobile phone companies have been claiming the safety of the wireless communication devices. The only possible adverse biological effect of radio frequency transmission they conceded was localized body heating. This is despite for example a team of scientists funded by Telstra in Australia who investigated links between cellular phones and cancer and turned up significant finding of adverse health effects. The study was rejected on the grounds that publication would cause a panic. In 1997, a publication in Radiation Research [26] suggested that exposure to radio frequency radiation may increase lymphoma incidence in mice and ignited the controversy. Along with the fear for the use of wireless receivers, the mobile phone base stations presented an even greater source of apprehension. This is despite the consensus of the scientific community that the power from the mobile phone base station antennas is far too low to produce health hazards, as long as people are kept away from direct access to the antennas. A large body of research was conducted, including a 1999 review [18] and a 2005 review [19]. Although some scientific evaluation showed that there is an unconvincing link between radio frequency exposure and cancer, a number of recent experimental studies show mobile phone radiation causes changes in gene expression and human cells [8], [12], [22]. In a recent revelation, the INTERPHONE study [11] announced a number of conclusions based on an interview-based case-control study conducted in 13 countries. We review the announcement in the next section.

Another concern is the growing development of wireless networks in the infrastructures of cities and towns. An upcoming technology is the Wireless Mesh Networking which seeks to cover entire areas with wireless communications. In July 2004, the city of Philadelphia in the United States announced the Wireless Philadelphia Project [31]. Such project is assured to be followed by others, with research conducted to develop the technology to englobe all living space with wireless communications capabilities. These ever increasing chances of prolonged and constant exposure to radio frequency radiation present the challenging question of health and safety.

\section{THE INTERPHONE PROJECT}

INTERPHONE is a multi-national project testing whether using mobile phones increases the risk of various cancers of the head and neck. The project comprises national epidemiological studies from 13 countries, which are coordinated by the International Agency for Research on Cancer (IARC), an agency of the World Health Organization (WHO) [11]. The International Journal of Epidemiology published a combined data analysis from a multi national population-based casecontrol study of glioma and meningioma, the most common types of brain tumour [13]. This is the first in a series of combined data analyses of head and neck tumours published as part of the internationally coordinated INTERPHONE project. The authors reported the following conclusion [7]:

- Overall, no increase in risk of glioma or meningioma was observed with use of mobile phones. There were suggestions of an increased risk of glioma at the highest exposure levels, but biases and error prevent a causal interpretation. The possible effects of long-term heavy use of mobile phones require further investigation.

- In the press release accompanying the release of the paper, Dr Christopher Wild, Director of the International Agency for Research on Cancer (IARC) said: "An increased risk of brain cancer is not established from the data from Interphone. However, observations at the highest level of cumulative call time and the changing patterns of mobile phone use since the period studied by Interphone, particularly in young people, mean that further investigation of mobile phone use and brain cancer risk is merited."

- Commenting on the study, Michael Milligan, Secretary General of the Mobile Manufacturers Forum said "The INTERPHONE project is the biggest study of its kind ever undertaken in this field and provides significant further reassurance about the safety of mobile phones. The overall analysis is consistent with previous studies and the significant body of research, reporting no increased health risk from using mobile phones."

- He continued "The absence of increased health risks include long term mobile phone use for more than 10 years. 
The authors make it clear that the data was insufficient for a clear interpretation of possible risk from self-reported heavy use due to a number of possible errors or biases. For example, the paper notes that there is evidence that people diagnosed with a brain tumour over-reported their past mobile phone use and that recall bias-like this may be more likely if subjects perceive that mobile phone use is associated with brain tumours, as has been widely speculated in the media."

- "Mobile phone users can take comfort in the fact that there is already a substantial body of scientific evidence on the long-tem use of mobile phones through wholeof-life animal studies, which have found no link between long-term exposure to radiofrequency and health impacts," added Mr Milligan.

- The INTERPHONE results now need to be considered by independent health authorities, such as the World Health Organization (WHO) and other expert groups to assess their significance, if any, to people's health.

- Mr Milligan added "The mobile industry supports the need for ongoing research. In fact, a number of longerterm studies are already underway such as the COSMOS study, which will follow the health of 250,000 European mobile users for 20-30 years, and several studies are now looking at children and teenagers, including the international MOBI-kids and CEFALO studies and the Australian MoRPhEUS project."

- Although INTERPHONE is a large and important study, it must be viewed in context as only one of many studies that will be used in the overall cancer-risk assessment to be undertaken by IARC in 2011 .

- The mobile phone industry takes all questions regarding the safety of mobile phones seriously and has a strong commitment to supporting ongoing scientific research such as the way it supported the INTERPHONE project.

- This study is part of the combined analysis of the national data collected as part of the 13 country INTERPHONE project, coordinated by the International Agency for Research on Cancer (IARC).

- The MMF provided partial funding for the INTERPHONE project in conjunction with the GSM Association, the European Commission and many national research funding bodies. Funding was provided in such a manner as to ensure the full scientific independence of the study and the terms of the funding agreement are publicly available at http://www.iarc.fr/en/researchgroups/RAD/RCAd.html.

- Tumours of the nervous system are rare and account for less than $2 \%$ of all malignancies (about 175,000 cases per year worldwide). Gliomas are a type of brain tumour arising in cells of the brain and are diagnosed each year at 6-8 per 100,000 people in the west. Meningiomas arise from cells that make up the covering around the brain and are even rarer, affecting fewer than 2 per 100,000 people.

The key message of the article is "INTERPHONE is the largest case-control study of mobile phone use and brain tumours yet and includes the largest numbers of users with at least 10 years of exposure. A reduced OR for glioma and meningioma related to ever having been a regular mobile phone user possibly reflects participation bias or other methodological limitations. No elevated OR for glioma or meningioma was observed $\geq 10$ years after first phone use. There were suggestions of an increased risk of glioma, and much less so meningioma, in the highest decile of cumulative call time, in subjects who reported usual phone use on the same side of the head as their tumour and, for glioma, for tumours in the temporal lobe. Biases and errors limit the strength of the conclusions that can be drawn from these analyses and prevent a causal interpretation" [13]. The news of the report was received with different perceptions. Phone and electronic companies saw the news in support of the safety of the technology. Some media reported it as a warning and a need to investigate further [1].

Increasingly, populations are growing weary of possible harmful effect by radio frequency radiation; be it the use of mobile phones, a phone company wanting to build a new phone tower or an incidence of a large number of cancer cases by employees of a media company. Whether radio frequency radiation is harmful or not, there is no doubt that studies of links between electromagnetic fields and cancer and other health failures will take place at an increasing pace, both in numbers and depth of scrutiny. While some of the studies will be in vitro, conducted mostly by medically oriented researchers, others will rely on statistical studies to distinguish patterns. Such studies would need data currently not available.

\section{EMF POLLUtion MEASURING}

There have been relatively few attempts at measuring EMF pollution. Often, it was done in a restricted context, within a research problem, to prove for example its negative effect. Balmori and Hallberg (2007) [4] support the hypothesis that electromagnetic signals are associated with the decline in the sparrow population. They conduct a sampling procedure for the mean electric field strength and relate it to a count of sparrow at different locations in a region in Spain. Before that, measurement of radio frequency electric and magnetic fields was conducted by Mantiply et al. (1997) [16]. The authors use an attempt at a graphical representation. In general, whenever there is a study on the subject, see Balmori (2006) [3] for a review, there is some form of measurement that takes place.

There is an increase in awareness of the possible dangers of EMF pollution. At least, there is awareness of the potential markets that may exploit the fear of such dangers. Small enterprizes are setting up systems that can monitor EMF pollution in a small area. Some companies seem to offer solutions. Activities are also sparked by governments in some countries willing to support research in this area [9]. There is definitely the need for the creation of databases that record EMF pollution levels and to present that data in Geographic Information Systems (GIS) format. For example Paolino et al. (2001) [23] presents a GIS-based visual approach to the problem. But overall, there is no organized effort in most countries to collect the data. This could possibly be due to the sensitivities associated with the topic. EMF are used in a lot of the technical innovations and are part of progress. The cost 
of stopping the technological advances could be high for some economies. As long as there is no clear link to catastrophic health issues, the probabilities of danger are downplayed.

We propose the idea of setting up a database that is free of subjective influence. Its design is made to ensure access to data by all researchers and the public. The conclusions reached by the users of the database would be their own. The database purpose is to provide reliable information. In the next sections, we discuss associated issues and methodology.

\section{Frequency Detection And Capture}

Spectrum analysis allows the monitoring of various RF signatures. The frequencies can be monitored through the use of spectrum estimators. These devices assists the identification of the frequencies within a range and plot them against the respective amplitude [2]. In considering a novel dynamic sensor network architecture for detecting and mapping EMF, individual measurement sensors are permitted to be mobile. The measurement sensors are randomly distributed and sensors within a range of each other are networked and clustered under an anchor sensor node. This ensures that smaller sensors with smaller coverage footprint and power resource can be used. Similarly, mobile sensors can change domain of control when they move away outside the range of anchor control. The role of the anchor node is to forward measurements from sensors under its domain of control. This architecture ensures that the server is not overwhelmed by many sensors trying to forward periodic measurements. It receives reports from only few anchor nodes. It also ensures scalability in terms of increasing the number of clusters as the mapping field is widened. The anchor nodes are provided with more resources such as more storage space, higher power regime and coverage range. The server is responsible for the processing and visual representation as well as long term storage and archiving of sensor measurements. It also provides the web access from remote users of the sensor maps.

The mapping infrastructure consists of three components: static RF sensors, mobile RF sensors and remote servers. The static sensors will monitor the RF power reading for a small coverage area periodically, store locally or forward the same to the server. The static and mobile sensors report their RF power measurements to the server using periodic wireless calls with the measurement values as content. The RF mapping sensors to be used will initially be off the shelf and ready to use devices such as radio receivers, cell phone receivers, wireless LAN transceivers, Bluetooth and ultra wideband receivers. They will be chosen to cover the spectral range from high frequency (HF), UHF, SHF, cellular phone bands from $400 \mathrm{MHz}$ to $2100 \mathrm{MHz}$ and up to the $\mathrm{X}$ band. This band therefore includes wireless networks, freeto-air and cable TV bands and ultra wideband. The intention is to use traditional receivers in these bands including cell phones, PDAs and laptops equipped with 802.11 access points. Where necessary, miniaturized transceivers or sensors can be developed and deployed as vehicle area networks, mounted on both fixed and mobile receivers. The fixed receivers will be used to map a selected site (eg. an industrial area). The mobile RF sensors will include mobile phones and will be carried by both pedestrians and by vehicles within a selected suburb. Each sensor will capture RF power and telemeter the measurement to a central receiver located in a laboratory. Where telemetering is impossible, the sensor will hold the measured information in a storage disc for downloading at the central receiver. Since the sensors sample the power spectrum for different sections of the electromagnetic spectrum, a twodimensional (2D) and three-dimensional (3D) power mapping of the area of choice will be undertaken. Each 2D mapping presents a summary of the power density recording for a particular type of radio signal propagation (eg. HF radio or the GSM frequency band). The 3D mapping is a layered view. Each layer is a 2D map of a particular RF service type. Presentation of the $2 \mathrm{D}$ and $3 \mathrm{D}$ maps will require a visual representation. In the next section, we elaborate on software engineering issues concerning the visual representation in real time of 3D electromagnetic fields mapping of selected areas and the dissemination of the information through various means and levels of access. Some of the concepts are taken from Pederson (2003) [24], where they can be found in more details.

\section{Software ENGINEERING}

This section discusses theoretical aspects in Software Engineering. It highlights important problems in visualization of data, when building a virtual representation of a physical world. In the case of a RF pollution mapping database, the data are to be reliable and considered objective. However, it is inevitable that visualization of information occurs. In fact, it is a desirable feature when large amounts of data are considered. In the context of a serious topic such as RF pollution, a severe display in the case of non-alarming data is a false positive that should be avoided. On the other hand, serious concentration could appear to be light if its representation is not 'adequate'. The data are hard, objective information to be used in scientific studies, most likely involving statistical analysis. But visualization has a subjective aspect, when it comes to bridging the gap between reality and its virtual representation. In this section, we discuss some of the issues associated with the physical-virtual association.

\section{A. Physical-Virtual Dichotomy in a Virtual Model of Infras- tructure Systems}

An ideal visual representation based on computer technology that allows cognitive perception and effective analysis of system infrastructure behavior should be characterized by attributes that do not distract human agents from their most absorbing and important routines. This is a widely shared opinion among designers of software intensive systems. The aims and objectives of a predominately ambient interaction process is to minimize the spatio-temporal and techno-cognitive encumbrance on users of the system by offering interface services, facilities and features that are user-centric, proactive, adaptive, automatic, understandable, flexible and not constrained to a physical place. Modeling and designing working infrastructure environments towards that end goal is a valuable exercise. Of 

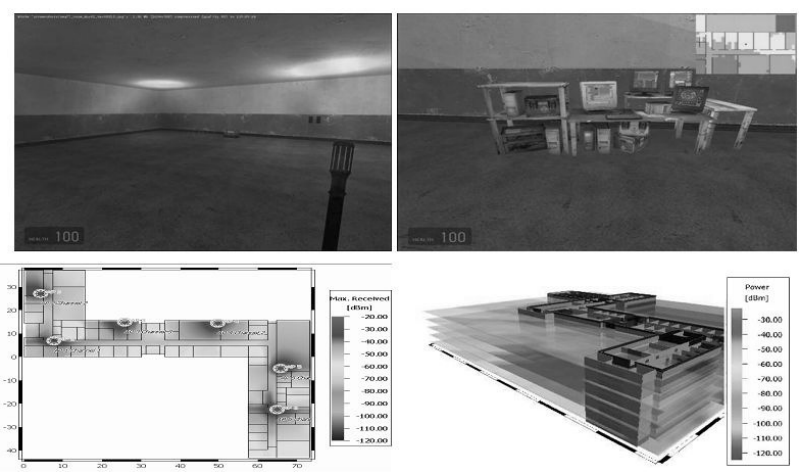

Fig. 3. Visual representation of RF radiation indoor with use of special 3D graphic effects in a single room (fog or mist), single floor and multi-floor visualisation map (WinProp application).

the four complementary focus areas mentioned above (adaptive; proactive; automatic; understandable) the work initially would involve taking the automation approach first. If we consider that in later stages of the research the additional aims would involve optimization of operational costs. Ultimately, the approach would be both user centric and based on an unbiased perspective on the physical and the virtual world.

1) Synchronizing the Two Worlds: The proposed approach for bridging the physical-virtual environment divide follows two complementary paths: (1) maximising redundancy so that activity-relevant object attributes in the two worlds are presented and preferably also manipulability in both worlds, (2) keeping these redundant attributes synchronised with minimal human attention.

2) Visual Representation of the Two Worlds: Based on 3D vector databases with planar objects it can be made possible to compute and provide mapping and visual representation of propagation, reflection, diffraction and shadowing of RF propagation for both indoor and outdoor scenarios. Different combinations of propagation model scenarios available can be divided into two main categories: (1) Empirical Models (i.e. One Slope Models, Motley Keenan Models, COST 231 Multi Wall Model) and (2) Ray Optical Propagation Models (i.e. Dominant Path Prediction Model, 3D Standard Ray Tracing (SRT), 3D Intelligent Ray Tracing (IRT) ). The visualisation application may include both multi floor buildings and outdoor projections. If multilevel calculation are required for each floor of a building computation may involve an arbitrary number of heights and can display the result in a $3 \mathrm{D}$ views. Thus, interference between floors and coverage problems can be easily eliminated. Figures 3 and 4 are examples of both indoor (multi floor building) and outdoor RF signal propagation visualization.

3) Building Objects and Databases of the Two Worlds: Mapping propagation and energy of RF signals in various environments requires detailed information about the geography, 3D topology and ecology (habitats and architectures) of the monitored space. To make results as much accurate as it is possible we would require to build 4D (space and time) vector databases for its RF propagation models. Moreover, we would require to include an additional techniques (meshups) and tools specially designed for building GIS databases that are augmented by models of signal propagation processes. For most outdoor and indoor planning tools, the handling of the GIS data would initially constitute the most critical part allowing the generation of building databases within a few minutes based on scanned bitmaps or CAD data. Combination of suitable GIS data and maps (Google maps) would allow the generation and storage of mesh-up artefacts.

In the context of RF pollution mapping, several visual representation models can be adopted. Figures 5-7 show examples of visual representations.

\section{B. Wireless Sensor Network Technology and RF Pollution}

Proliferation and increasing density of various mobile and stationary computing as communication devices could pose a potential threat to human health by forming various hot spots and transitory RF pathways. However, recent developments in computing and Wireless Sensor Technology (WSN), apart of itself representing a possible treat, could help in monitoring and preventing harmful RF pollution. This can be realised by aiding environmental RF pollution management with sensor networks and software systems that would allow a real-time monitoring of the environment, creation of visual mappings of RF energy distribution, dynamic reconfiguration of RF traffic (routing) and possible removal of high level RF energy clusters. This idea is presented in various simulation that use dedicated Single Value Decomposition (SVD) based computation algorithms [6].

\section{Gestalt Relations and Visualization of RF Pollution}

In early twenties of the last century, Wertheimer (1924) [29] formulated an original theory of visual gestalt experiences. The German word gestalt roughly translates to "whole" or "form". Wertheimer postulated that gestalten (or sensible structures) are actually perceived first and their constituent parts second only. Gestalt experiences can be perceived as connecting glue that make the universe whole. Accordingly, the boundary between a model of the system under observation and the rest of reality is like the boundary from one moment of time to the next. It is real and significant, yet transitory. If there were no boundaries of perception/view there would be no observable specifics. System boundaries can then be seen more in terms of pathways rather than constraints. By their nature, they may lead to future events, new discoveries, experiences and transitions that may relate to other subsystems, components and users; at other times and in various places. It is argued that in the gestalt approach in order to interpret what is perceived
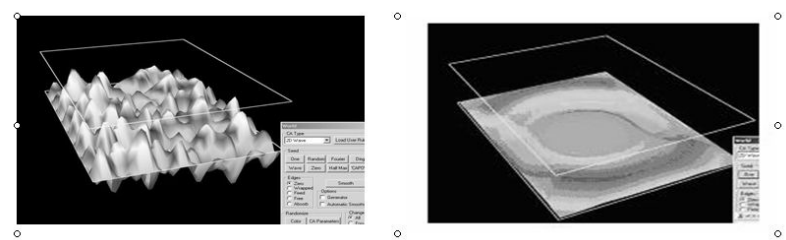

Fig. 4. Examples of outdoor single visual representation (Capow2007 application [27]). 


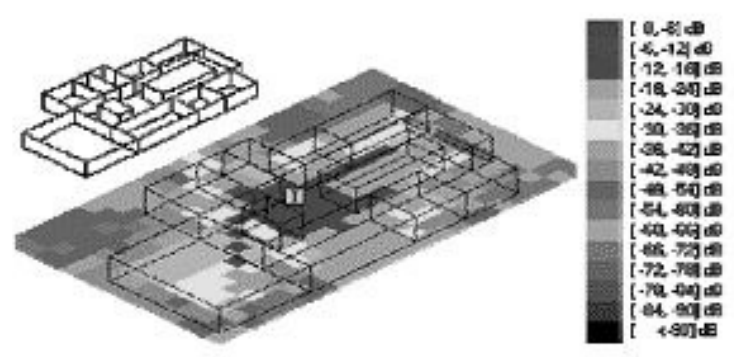

Fig. 5. Visual representation, CINDOOR, RF Coverage [15].

by our senses, we need to organize the information into certain groups or classes. This allows the information to be processed completely and without wasteful repetition. As an example, when we see a single area or a single object, it is perceived as such, but when fifteen objects are seen together, we aim to associate them by saying there is a constellation of objects/areas. Similarly, object's yawing, rolling or pitching motions depicted in time represent trajectories of its movements. Without the ability to group our visuals, that same configuration that would have appeared as: "area, area, ..., area" would take much longer to perceive and process the information thus limiting the observer's cognitive ability.

Many researchers have considered the gestalt theory as a being of a revolutionary nature which gave foundations for the Kuhnian visual representation models (Kuhn 1972, [14]). The Kuhnian approach highlights aspects of social negotiation of a meaning among community of users within a paradigm, and that any interpretation of observation is integral and inseparably intertwined with the active paradigm. Recent research into the perception of models, abstracts, representations and design compositions often refers to the gestalt aesthetics or rules and still remains a fertile ground for research. Aesthetics of gestalt influences the imagination of many researchers by generating new issues and solutions in the mainstream research of sensory gestalt. The gestalt theory should not be seen, however, as being limited to artificial two-dimensional abstract phenomena. The three-dimensional gestalt phenomena established under experimental conditions turned out to be difficult to explain within traditional gestalt theory as many failed attempts have proved [17] although, it was Gibson

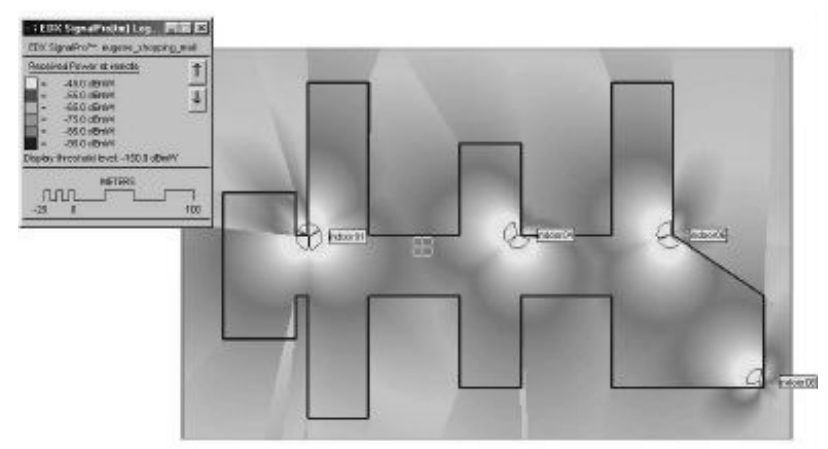

Fig. 6. Visual representation, EDX Signal Pro [15].

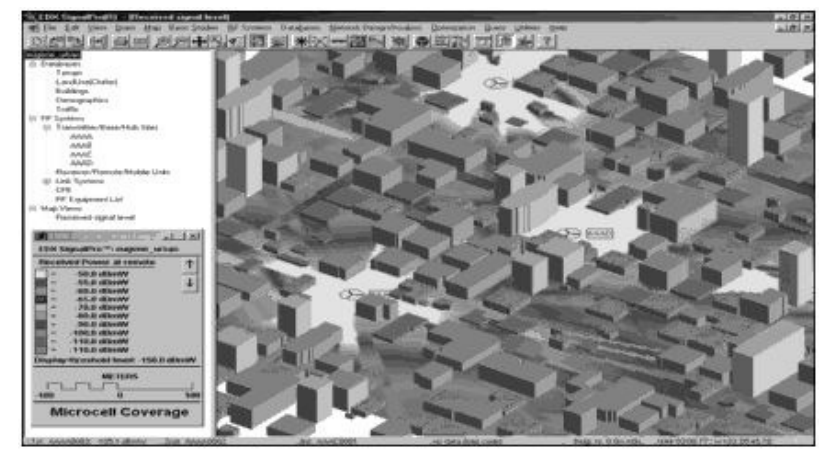

Fig. 7. Visual representation, EDX Signal Pro [15].

(1979) [10] with his theory of invariant optical structures who found a suitable solution for the problem . Gibson explained the experience of three-dimensional gestalten as perceptions of optical invariants. This was further developed into the ecological approach to a visual perception where remnants of the gestalt aesthetic tradition can still be found. It seems that perception of optical invariants can be viewed as a set of perceptual preferences; and affordances interpreted as aesthetic translations [10]. The theory of direct perception is considered as a theory of perceptual preferences for formal gestalten. The Wertheimer's gestalt-aesthetic tradition plays a significant role in this research and investigations not only because of human perceptual and aesthetic preferences but also because of the fact that a successful construction of large visual constructs requires an organization. This is vital for finding order, bounds and cognitive elements in both virtual and physical infrastructure models.

All of these issues relate to the visualization problem associated with a RF pollution mapping system. While we do not discuss these issues, we point to the necessity of addressing the subjective aspect inherent in any visualization of the RF radiation. Software engineering theory addresses issues in building a virtual representation of the real world.

\section{A DATA Algorithmic BACK-END}

Data Mining is an analytic process designed to explore large amounts of data. For example, Vector Machines have a strong theoretical background. They have proved to be successful in many classification problems and there is no statistical modeling in support Vector Machines. In classification, they offer an optimal solution, given that a particular objective function is maximized and the optimal solution exist. The popularity of support Vector Machines is that they offer a computationally efficient programming solution. In addition, they offer the "Black-Box" effect, that is they are a mathematical solution that does not require any modeling of the actual problem. It is all data driven. In the context of separating RF pollution data, this exercise may be essential for searching through a large database. Rather than build a model, which in the case of health studies may create controversy, data mining techniques rely on the concept of "Black Box" and mathematical optimization. While searching for a particular pattern, the user can specify characteristics of the pattern, 
leaving it to the software to search the database. We propose that a software solution that makes full use of the information in the database using data mining algorithms be attached to the database as a back-end.

\section{CONCLUSION}

Wireless sensor networks is a rapidly evolving technology. Applications of great significance can be achieved through the deployment of networks of sensor nodes that are cheap to manufacture but efficient and precise in their data collection. In the context of radio frequency radiation mapping, the architecture of wireless sensor networks can be designed to optimize the detection of radiation over industrial, commercial and populated zones. We propose the idea for a database to ensure access to EMF pollution data. The impartiality of the process is built into the database, although the visualization of information requires the sorting of issues associated with the world physical-virtual dual aspects. While the impartiality aspect is clear and common in most databases, it is of particular significance in the case of radio frequency pollution where the associated controversial issues may have hindered efforts to build such databases. A software engineering solution must be tailored to the problem along with the front-end data collection and back-end data information extraction and analysis.

\section{ACKNOWLEDGMENT}

The authors would like to thank Tim Aubrey and the Centre for Real-time Information Networks at the University of Technology Sydney for their support of this work.

\section{REFERENCES}

1] ABC - Australian Broadcasting Corporation, "Study probes link between cancer and phones," Reporter: Ticky Fullerton, 2010, broadcast: 17/05/2010. [Online]. Available: http://www.abc.net.au/ lateline/content/2010/s2902097.htm

[2] J. Agbinya, V. Lee, K. Aboura, and Z. Chaczko, "Recognition of radio signal pollution levels and signatures," in Proc. of the Fourth International Conference on Broadband Communication Information Technology and Biomedical Applications, Wroclaw, Poland, 2009.

[3] A. Balmori, "The incidence of electromagnetic pollution on the amphibian decline: Is this an important piece of the puzzle ?" Toxicological \& Environmental Chemistry, vol. 88, no. 2, pp. 287-299, Apr. - June 2006.

[4] A. Balmori and O. Hallberg, "The urban decline of the house sparrow (passer domesticus): a possible link with electromagnetic radiation," Electromagnetic Biology and Medicine, vol. 26, pp. 141-151, June 2007.

[5] E. W. Campion, "Power lines, cancer and fear," The New England Journal of Medicine, vol. 337, no. 1, pp. 44-46, 1997, editorial.

[6] Z. Chaczko and R. Klempous, "Anticipatory biomimetic middleware," in Proc. of the 9th International Conference on Computing Anticipatory Systems, Liege, Belgium, August 3-8, 2009.

[7] Disabled World, "Biggest study ever reports on mobiles and brain cancer," 2010. [Online]. Available: http://www.disabled-world.com/ health/cancer/cell-phone-cancer.php

[8] O. Erogul, E. Oztas, I. Yildirim, T. Kir, E. Aydur, G. Komesli, H. C. Irkilata, M. K. Irmak, and A. F. Peker, "Effects of electromagnetic radiation from a cellular phone on human sperm motility: an in vitro study," Archives of Medical Research, vol. 37, no. 7, pp. 840-843, Oct. 2006.

[9] P. Getsov, D. Teodosiev, E. Roumenina, M. Israel, G. Mardirossian, G. Sotirov, B. Srebrov, S. Velkoski, P. Gajesek, and D. Simunic, "Methods for monitoring electromagnetic pollution in the western balkan environment," in Proc of the Third Scientific Conference with International Participation, Space, Ecology, Nanotechnology, Safety (SENS 2007), Varna, Bulgaria, 2007.
[10] J. J. Gibson, The ecological approach to visual perception. Hillsdale, New Jersey: Lawrence Erlbaum Associates, 1986.

[11] GSMA, "INTERPHONE Project Overview," 2009. [Online]. Available: http://www.gmsworld.com/documents/health/research/ GSMA_INTERPHONE_Overview.pdf

[12] L. Hardell, K. H. Mild, M. Carlberg, and F. Soderqvist, "Tumour risk associated with use of cellular telephones or cordless desktop telephones," World Journal of Surgical Oncology, vol. 4, p. 74, Oct. 2006.

[13] INTERPHONE Study Group, "Brain tumour risk in relation to mobile telephone use: results of the INTERPHONE international case-control study," International Journal of Epidemiology, pp. 1-20, May 17, 2010, doi:10.1093/ije/dyq079, advance access.

[14] T. S. Kuhn, The structure of scientific revolutions, 3rd ed. Chicago: The University of Chicago Press, 1996.

[15] P. Madej, "3D wireless networks simulator - visualization of radio frequency propagation for WLANs," Master's thesis, University of Dublin Trinity College, Dublin, Ireland, 2006. [Online]. Available: http://www.scss.tcd.ie/publications/tech-reports/ reports.07/TCD-CS-2007-15.pdf

[16] E. D. Mantiply, K. R. Pohl, S. W. Poppell, and J. A. Murphy, "Summary of measured radiofrequency electric and magnetic fields $(10 \mathrm{kHz}$ to $30 \mathrm{GHz}$ ) in the general and work environment," Bioelectromagnetics, vol. 18, pp. 563-577, 1997.

[17] W. Metzger, Gesetze des Sehens, 3rd ed. Frankfurt: Verlag Waldemar Kramer, 1975

[18] J. E. Moulder, L. S. Erdreich, R. S. Malyapa, J. Merritt, W. F. Pickard, and Vijayalaxmi, "Cell phones and cancer: What is the evidence for a connection?" Radiation Research, vol. 151, pp. 513-531, 1999.

[19] J. E. Moulder, K. R. Foster, L. S. Erdreich, and J. P. McNamee, "Mobile phones, mobile phone base stations and cancer: a review," International Journal of Radiation Biology, vol. 81, no. 3, pp. 189-203, Mar. 2005.

[20] NIEHS, "NIEHS REPORT on health effects from exposure to power-line frequency electric and magnetic fields," The National Institute of Environmental Health Sciences, 1999. [Online]. Available: http://www.niehs.nih.gov/health/docs/niehs-report.pdf

[21] NRPB, "ELF electromagnetic fields and the risk of cancer," Documents of the NRPB, vol. 12, no. 1, pp. 1-179, 2001.

[22] D. J. Panagopoulos, E. D. Chavdoula, I. P. Nezis, and L. H. Margaritis, "Cell death induced by GSM 900-MHz and DCS 1800-MHz mobile telephony radiation," Mutation Research, vol. 626, pp. 69-78, Jan. 2007. [Online]. Available: http://kyttariki.biol.uoa.gr/EMR-GROUP/ article-oct-2006.pdf

[23] L. Paolino, M. Sebillo, G. Tortora, and G. Vitiello, "Monitoring electromagnetic pollution: A GIS-based visual approach," in Proc. of the Second International Workshop on Multimedia Databases and Image Communication 2001, M. Tucci, Ed. Berlin Heidelberg: SpringerVerlag, Lecture Notes In Computer Science 2184, 2001, pp. 90-101.

[24] T. Pederson, "From conceptual links to causal relations - physical-virtual artefacts in mixed-reality space," Ph.D. dissertation, Dept. of Computing Science, Umea university, Umea, Swewden, 2003. [Online]. Available: http://umu.diva-portal.org/smash/record.jsf?pid=diva2:140782

[25] R. Pool, "Emf-cancer link still murky," Nature, vol. 349, Feb. 1991.

[26] M. H. Repacholi, A. Baten, V. Gebski, D. Noonan, J. Finnie, and A. W. Harris, "Lymphomas in Eu-Pim1 transgenic mice exposed to pulsed 900 MHz electromagnetic fields," Radiation Research, vol. 147, pp. 631-640, 1997.

[27] R. Rucker, "Cellular automata for electric power simulation," San Jose State University, San Jose, California, USA, 2007. [Online]. Available: http://www.cs.sjsu.edu/ rucker/capow/index.html

[28] P. K. Verkasalo, E. Pukkala, J. Kaprio, K. V. Heikkila, and M. Koskenvuo, "Magnetic fields of high voltage power lines and risk of cancer in finnish adults: nationwide cohort study," BMJ, vol. 313, pp. 1047-1051, Oct. 1996.

[29] M. Wertheimer, Uber Gestalttheorie (Gestalt Theory). Erlangen, 1924, the original translation by Willis D. Ellis published in Source Book of Gestalt Psychology, New York: Harcourt, Brace and Co., 1938, reprinted New York: Gestalt Journal Press, 1997.

[30] N. Wertheimer and E. Leeper, "Electrical wiring configurations and childhood cancer," American Journal of Epidemiology, vol. 109, no. 3, pp. 273-284, 1979.

[31] Wireless Philadelphia, "Wireless philadelphia business plan - wireless broadband as the foundation for a digital city," Report presented to the Honorable John F. Street, Mayor of the city of Philadelphia, by The Wireless Philadelphia Executive Committee, Feb. 9, 2005. 